\title{
A Cancellation Nanoflare Model for Solar Chromospheric and Coronal Heating. III. 3D Simulations and Atmospheric Response
}

\author{
P. Syntelis (i) and E. R. Priest (10) \\ St Andrews University, Mathematics Institute, St Andrews KY16 9SS, UK; ps84@st-andrews.ac.uk \\ Received 2019 December 9; revised 2020 January 24; accepted 2020 January 24; published 2020 March 3
}

\begin{abstract}
Inspired by recent observations suggesting that photospheric magnetic flux cancellation occurs much more frequently than previously thought, we analytically estimated the energy released from reconnection driven by photospheric flux cancellation, and propose that it can act as a mechanism for chromospheric and coronal heating. Using two-dimensional simulations we validated the analytical estimates and studied the resulting atmospheric response. In the present work, we set up 3D resistive MHD simulations of two canceling polarities in a stratified atmosphere with a horizontal external field to further validate and improve upon the analytical estimates. The computational evaluation of the parameters associated with the energy release are in good qualitative agreement with the analytical estimates. The computational Poynting energy flux into the current sheet is in good qualitative agreement with the analytical estimates, after correcting the analytical expression to better account for the horizontal extent of the current sheet. The atmospheric response to the cancellation is the formation of hot ejections, cool ejections, or a combination of both hot and cool ejections, which can appear with a time difference and/or be spatially offset, depending on the properties of the canceling region and the resulting height of the reconnection. Therefore, during the cancellation, a wide spectrum of ejections can be formed, which can account for the variety of multi-thermal ejections associated with Ellerman bombs, UV bursts, and IRIS bombs, and also other ejections associated with small-scale canceling regions and spicules.
\end{abstract}

Unified Astronomy Thesaurus concepts: Solar coronal heating (1989); Magnetohydrodynamics (1964); Solar magnetic reconnection (1504); Solar activity (1475); Magnetic fields (994); Magnetohydrodynamical simulations (1966); Solar coronal transients (312)

\section{Introduction}

Coronal heating is one of the biggest open questions in solar and stellar physics. Many ideas have been proposed to explain how the solar corona is heated to millions of degrees and the chromosphere to tens of thousands of degrees, with most models considering that the energy required originates either from waves (e.g., resonant absorption or phase mixing Klimchuk 2006; Parnell \& De Moortel 2012; Priest 2014) or from magnetic reconnection (e.g., nanoflares driven by photospheric motions Parker 1988; Priest et al. 2002). So far, no mechanism has conclusively been identified as the one heating the solar corona.

In recent years, observations of the solar photosphere and atmosphere have revealed that the cancellation of opposite magnetic polarities is a much more common process than previously thought. Magnetic flux cancellation is the process whereby two opposite polarities approach each other, interact via magnetic reconnection, and eventually submerge into the solar interior (e.g., Parker 1979; van Ballegooijen \& Martens 1989). The reconnection associated with flux cancellation has been long proposed as a mechanism for heating X-ray bright points (e.g., Priest et al. 1994; Parnell \& Priest 1995), but the great increase in frequency of cancellation events suggests that they may also be heating chromospheric and coronal plasma much more widely.

Observations of the plasma properties above cancellation regions have revealed that both cool and hot jets can be ejected above the canceling polarities as a result of the reconnection driven by the cancellation. Depending on the height where that reconnection occurs, the localized energy release and resulting plasma ejections may show up as Ellerman bombs, UV and
EUV bursts, Interface Region Imaging Spectrograph (IRIS) bombs, and other impulsive releases of energy (e.g., Watanabe et al. 2011; Vissers et al. 2013; Peter et al. 2014; Kim et al. 2015; Rutten et al. 2015; Rezaei \& Beck 2015; Vissers et al. 2015; Nelson et al. 2016, 2017; Reid et al. 2016; Rutten 2016; Tian et al. 2016, 2018; Hong et al. 2017; Libbrecht et al. 2017; van der Voort et al. 2017; Toriumi et al. 2017; Chen et al. 2019; Huang et al. 2019; Ulyanov et al. 2019). In addition to the above energy bursts and plasma ejections, recent observations have suggested that the cancellation of opposite polarities at the footpoints of coronal loops could be responsible for the brightening of coronal loops (Tiwari et al. 2014; Chitta et al. 2017a, 2018; Huang et al. 2018; Sahin et al. 2019).

State-of-the-art observations from the Sunrise balloon mission (Solanki et al. 2010, 2017) measured the photospheric magnetic field with a spatial resolution of 0 ." 15 , six times the resolution of the Helioseismic Imager on the Solar Dynamics Observatory. These novel observations revealed that the rate of magnetic flux cancellation is higher than previously thought by an order of magnitude (Smitha et al. 2017). These findings suggest that the energy released during these previously unseen canceling flux elements is more ubiquitous than previously realized.

Inspired by these observations, as a first step toward estimating the energy release, we analytically examined the cancellation of two opposite polarities (Priest et al. 2018, hereafter Paper I). By assuming that the two converging opposite polarities are in the presence of a uniform horizontal magnetic field, we estimated the energy released during the reconnection driven by the cancellation, and the height of release. Our analysis led us to propose that reconnection driven 
by the photospheric flux cancellation is a nanoflare-based mechanism able to heat the solar chromosphere and corona.

The next step in developing the model was to set up twodimensional (2D) simulations of flux cancellation that have the same features as our analytical model (Syntelis et al. 2019, hereafter Paper II), so that they may be compared. By assuming two converging opposite magnetic polarities inside an overlying uniform horizontal magnetic field, we compared the analytical expressions with the simulations. We found that our analytical expressions accurately estimated the characteristic parameters associated with the reconnection region, such as the magnitude of the inflowing magnetic field and velocity, the length of the current sheet, and the resulting energy converted to heat. In addition, because the simulations included a stratified atmosphere, we were able to study the atmospheric response to the reconnection occurring at different heights. We found that depending on the initial height of the null point, the cancellation-driven reconnection could produce either hot or cool ejections or a combination of both hot and cool ejections, in a manner similar to what it seen in observations.

In this paper, we take the next step toward developing our model by setting up 3D simulations of reconnection driven by flux cancellation. To do so, we consider two converging opposite polarities in the presence of an overlying horizontal magnetic field; a field similar to that of Paper I. We again include a stratified atmosphere, to compare with the $2 \mathrm{D}$ simulations.

The paper is organized as follows. In Section 2 we summarize the analytical theory presented in Paper I. In Section 3 we describe our computational model, compare it with the analytical theory, and examine the atmospheric response. In Section 4 we discuss our results.

\section{Summary of Theory for Energy Release Driven by Photospheric Flux Cancellation in 3D}

Here we summarize the theoretical estimates of the energy release by steady-state magnetic reconnection driven by flux cancellation in three dimensions, as presented in Paper I.

\subsection{Energy Conversion during Photospheric Flux Cancellation in a Horizontal Field}

\subsubsection{Magnetic Configuration}

At the photosphere, we considered two sources, one with positive and one with negative magnetic flux $( \pm F)$, situated at points $\mathrm{B}(d, 0,0)$ and $\mathrm{A}(-d, 0,0)$ on the $x$-axis. In the atmosphere, we included a uniform and horizontal magnetic field $B_{0} \hat{x}$. For simplicity, we assumed that the sources have equal flux and that they were aligned with the overlying field (see Paper I for discussion on more general configurations). The resulting magnetic field is given by

$$
\boldsymbol{B}=\frac{F}{2 \pi} \frac{\hat{\boldsymbol{r}}_{1}}{\boldsymbol{r}_{1}^{2}}-\frac{F}{2 \pi} \frac{\hat{\boldsymbol{r}_{2}}}{\boldsymbol{r}_{2}^{2}}+\boldsymbol{B}_{0} \hat{\boldsymbol{x}},
$$

where

$$
\boldsymbol{r}_{1}=(x-d) \hat{\boldsymbol{x}}+\boldsymbol{y} \hat{\boldsymbol{y}}+z \hat{\boldsymbol{z}}, \quad \boldsymbol{r}_{2}=(x+d) \hat{\boldsymbol{x}}+\boldsymbol{y} \hat{\boldsymbol{y}}+z \hat{z},
$$

are the vector distances from the two sources to a point $P(x$, $y, z)$.

The magnetic field is non-dimensionalized with respect to $B_{0}$, and the length with respect to the interaction distance
(Longcope 1998)

$$
d_{0}=\left(\frac{F}{\pi B_{0}}\right)^{1 / 2},
$$

so we define dimensionless quantities:

$$
\begin{aligned}
\bar{B}_{x} & =\frac{B_{x}}{B_{0}}, \bar{d}=\frac{d}{d_{0}}, \bar{r}=\frac{r}{d_{0}}, \\
\bar{x} & =\frac{x}{d_{0}}, \bar{y}=\frac{y}{d_{0}}, \bar{z}=\frac{z}{d_{0}} .
\end{aligned}
$$

The $x$-component of the field then becomes

$$
\bar{B}_{x}=\frac{\bar{x}-\bar{d}}{2 \bar{r}_{1}^{3}}-\frac{\bar{x}+\bar{d}}{2 \bar{r}_{2}^{3}}+1,
$$

Along the $z$-axis at $\bar{x}=\bar{y}=0$, the $y$ - and $z$-components of the magnetic field therefore vanish $\left(\bar{B}_{y}=\bar{B}_{z}=0\right)$, while the $x$ component becomes

$$
\bar{B}_{x}=-\frac{\bar{d}}{\left(\bar{d}^{2}+\bar{z}^{2}\right)^{3 / 2}}+1 .
$$

Suppose the two sources approach one another at speeds $\pm v_{0}$. The evolution of the magnetic field topology is described in detail in Paper I, and is schematically represented in Figure 1. The two sources are not magnetically linked when they are far away $(\bar{d}>1$, Figure $1(\mathrm{a}))$. When $\bar{d}=1$, a highorder null point appears at the origin (point N, Figure 1(b)). As the sources approach each other $(\bar{d}<1)$, a semicircular separator is formed in the $y z$-plane at $x=0$ (marked as $\mathrm{S}$, Figure $1(\mathrm{c}))$. The radius $\left(\bar{z}_{S}\right)$ of the separator is found from Equation (5) by setting $\bar{B}_{x}=0$, namely,

$$
\bar{z}_{S}^{2}=\bar{d}^{2 / 3}-\bar{d}^{2},
$$

and $z_{S}$ is plotted in Figure 4(a) of Paper I. As the sources approach, the radius of the separator increases to a maximum of $\left(\bar{z}_{s}\right)_{\max }=(4 / 27)^{1 / 4}$ at $\bar{d}=1 / 3^{3 / 4}$ and then drops to zero as $\bar{d}$ tends to zero.

\subsubsection{Inflow Plasma Speed $\left(\mathrm{v}_{\mathrm{i}}\right)$ and Magnetic Field $\left(\mathrm{B}_{\mathrm{i}}\right)$ at the Reconnection Region}

During the reconnection driven by flux cancellation, a semiannular current sheet is formed at the location of the separator. In the $x z$-plane, this corresponds to a sheet of length $L$ (Figure 1(d)). By linearizing the field around the current sheet, the magnetic field strength of the plasma flowing into the current sheet is found to be

$$
\bar{B}_{i}=\frac{3\left(1-\bar{d}^{4 / 3}\right)^{1 / 2}}{2 \bar{d}^{1 / 3}} \bar{L}
$$

The speed $\left(v_{i}\right)$ of the plasma flowing into the current sheet is derived by estimating the rate of change of flux below the current sheet, and is found to be

$$
\bar{v}_{i}=\frac{2}{3 \bar{L}} \bar{d}^{-1 / 3},
$$

where $v_{i}$ is normalized with respect to $v_{0}$.

\subsubsection{Energy Release}

The energy release follows by estimating the Poynting flux flow into the current sheet. The Poynting influx from both sides 


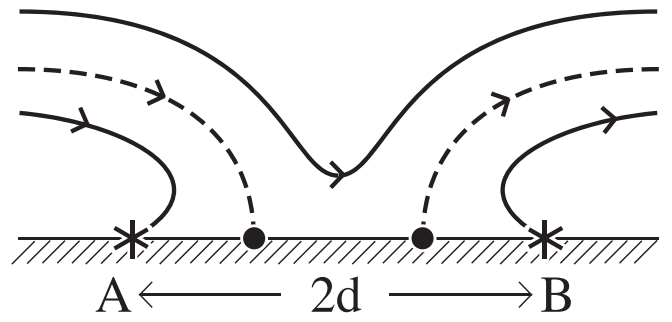

(a) $d>d_{o}$

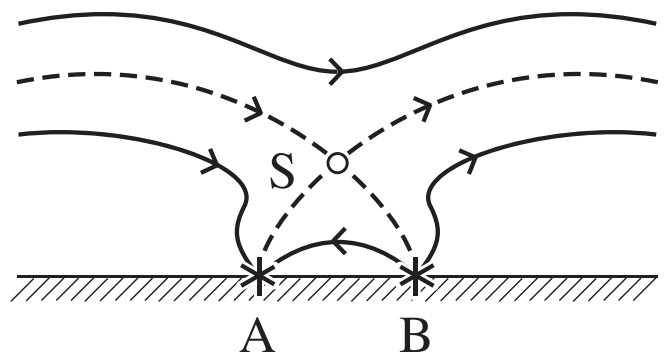

(c) $\mathrm{d}<\mathrm{d}_{\mathrm{o}}$

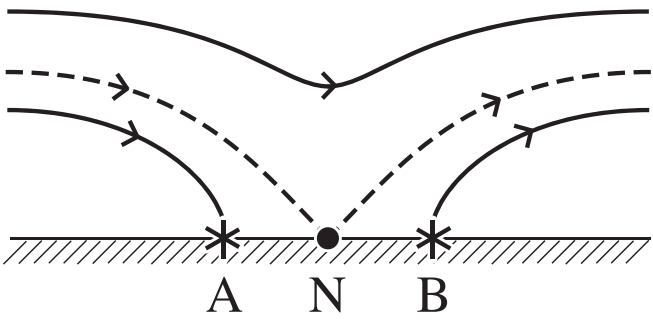

(b) $d=d_{o}$

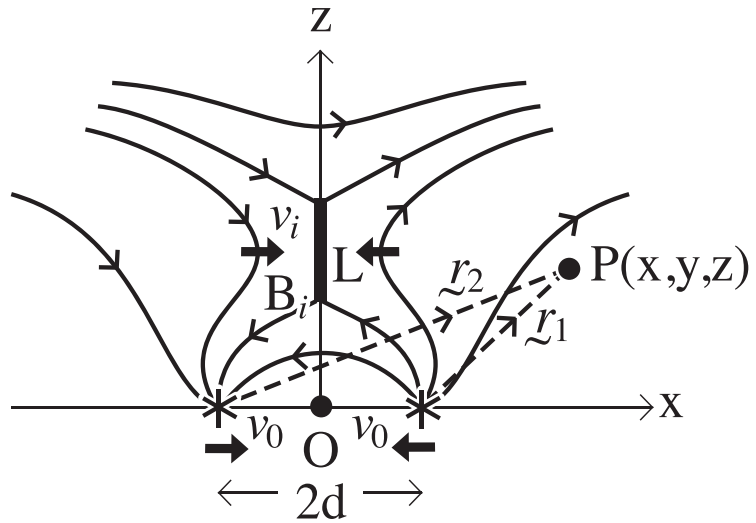

(d) $d=0$

Figure 1. Magnetic topology in the $x z$-plane during reconnection driven by photospheric flux cancellation when (a) $\bar{d} \equiv d / d_{0}>1$, (b) $\bar{d}=1$, and (c) $\bar{d}<1$. (d) Schematic showing the notation used to describe the reconnection region. The figure is taken from Paper I.

of the current sheet of length $L$ is

$$
S_{i}=2 \frac{v_{i} B_{i}^{2}}{\mu} L \pi z_{s} .
$$

The length of the current sheet depends on the type of reconnection. In the case with fast reconnection, $L$ is determined by assuming that the inflow speed has a known Mach Alfvén number $\alpha\left(v_{i}=\alpha v_{A i}\right.$, where $v_{A i}=B_{i} / \sqrt{\mu \rho_{i}}$ and $\rho_{i}$ is the density of the inflowing plasma). Then, using Equation (7) and (8), $L$ becomes

$$
\frac{L^{2}}{d_{0}^{2}}=\frac{4 v_{0}}{9 \alpha v_{A 0}} \frac{1}{\left[1-\left(d / d_{0}\right)^{4 / 3}\right]^{1 / 2}},
$$

where

$$
v_{A 0}=\frac{B_{0}}{\sqrt{\mu \rho_{i}}},
$$

is a hybrid Alfvén speed. Then, from Equation (9), the Poynting flux into the current sheet for fast reconnection is

$$
\frac{\mu}{v_{0} B_{0}^{2} d_{0}^{2}} S_{i}=2 \frac{2 \pi}{3} \frac{M_{A 0}}{\alpha} \frac{\left[1-\bar{d}^{4 / 3}\right]}{\bar{d}^{2 / 3}} .
$$

Lastly, the energy release is derived by assuming that during fast reconnection, $\frac{2}{5}$ of the energy is converted to heat (Priest 2014):

$$
\frac{\mu}{v_{0} B_{0}^{2} d_{0}^{2}} \frac{d W}{d t}=0.8 \frac{2 \pi}{3} \frac{M_{A 0}}{\alpha} \frac{\left[1-\bar{d}^{4 / 3}\right]}{\bar{d}^{2 / 3}} .
$$

\section{Numerical Computations}

\subsection{Numerical Setup}

We numerically solve the 3D MHD equations in Cartesian geometry using the Lare3D code (v3.2) (Arber et al. 2001). The equations in dimensionless form are

$$
\begin{gathered}
\frac{\partial \rho}{\partial t}+\nabla \cdot(\rho \boldsymbol{v})=0 \\
\frac{\partial(\rho \boldsymbol{v})}{\partial t}=-\nabla \cdot(\rho \boldsymbol{v} \boldsymbol{v})+(\nabla \times \boldsymbol{B}) \times \boldsymbol{B}-\nabla P+\rho \boldsymbol{g} \\
\frac{\partial(\rho \epsilon)}{\partial t}=-\nabla \cdot(\rho \epsilon \boldsymbol{v})-P \nabla \cdot \boldsymbol{v}+Q_{\mathrm{j}}+Q_{\mathrm{v}}+Q_{c}, \\
\frac{\partial \boldsymbol{B}}{\partial t}=\nabla \times(\boldsymbol{v} \times \boldsymbol{B})-\nabla \times(\eta \nabla \times \boldsymbol{B}), \\
\epsilon=\frac{P}{(\gamma-1) \rho}, \\
P=\frac{\rho k_{B} T}{\mu_{m}},
\end{gathered}
$$

where $\rho, \boldsymbol{v}, \boldsymbol{B}$, and $P$ are density, velocity vector, magnetic field vector, and gas pressure. Gravity is $g_{0}=274 \mathrm{~m} \mathrm{~s}^{-1}$. Viscous heating $\left(Q_{\mathrm{v}}\right)$ and Joule dissipation $\left(Q_{\mathrm{j}}\right)$ are included. Heat conduction $\left(Q_{c}\right)$ is treated using super-time stepping (Meyer et al. 2012). We assume a perfect gas with a specific heat of $\gamma=5 / 3$. The reduced mass is $\mu_{m}=m_{f} m_{p}$, where $m_{p}$ is the mass of proton and $m_{f}=1.2$. $k_{\mathrm{B}}$ is the Boltzmann constant.

In the $2 \mathrm{D}$ simulations of Paper II, we adopted a non-uniform resistivity profile that was a function of the current density. The 


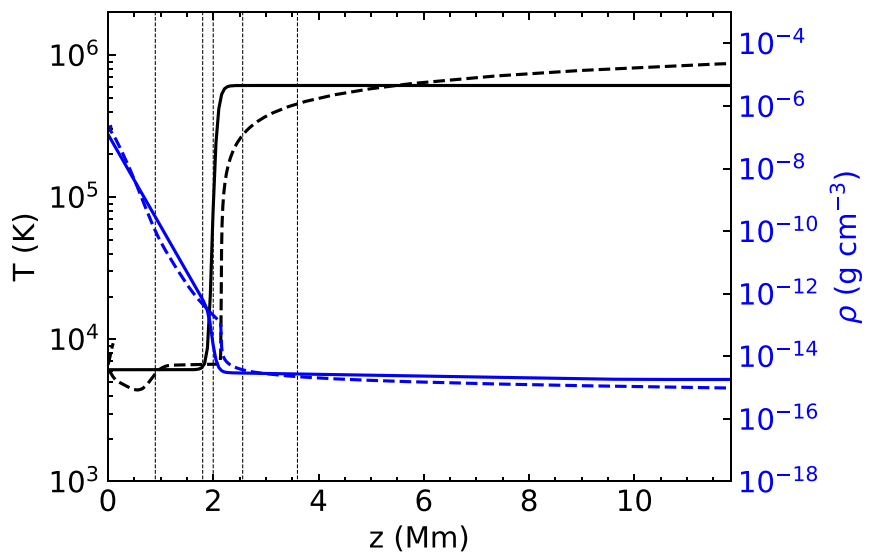

Figure 2. Atmospheric temperature (solid black) and density (solid blue). The dashed lines show the temperature and density of the 1D C7 model of Avrett \& Loeser (2008). The vertical dashed lines show the heights of the null point at $t=0$ for Cases $1-5$ of Table 1 (left to right, respectively).

functional form was selected to make the explicit resistivity larger than numerical diffusion. In the current 3D simulations, this is not possible, so the resolution is such that numerical diffusion dominates the reconnection region. Therefore, in this paper, we assume a low uniform explicit resistivity of $\eta=10^{-2}$ everywhere across the numerical domain and allow numerical diffusion to permit reconnection at the current sheet associated with flux cancellation. Exceptions to that are the boundaries, where $\eta$ decreases to zero to couple the field and flow properly there.

The normalization is based on photospheric values of density $\rho_{\mathrm{u}}=1.67 \times 10^{-7} \mathrm{~g} \mathrm{~cm}^{-3}$, length-scale $H_{\mathrm{u}}=180 \mathrm{~km}$ and magnetic field strength $B_{\mathrm{u}}=300 \mathrm{G}$. From these we obtain temperature $T_{\mathrm{u}}=6234 \mathrm{~K}$, pressure $P_{\mathrm{u}}=7.16 \times 10^{3} \mathrm{erg} \mathrm{cm}^{-3}$, velocity $v_{\mathrm{u}}=2.1 \mathrm{~km} \mathrm{~s}^{-1}$, and timescale $t_{\mathrm{u}}=86.9 \mathrm{~s}$.

The domain has a size of $x \in[-11.88,11.88] \mathrm{Mm}$ and $y \in$ $[-11.88,11.88] \mathrm{Mm}$ in the horizontal direction and $z \in[0$, $11.88] \mathrm{Mm}$ in the vertical direction, on a $440 \times 440 \times 220$ uniform grid. A hyperbolic tangent profile is used for the atmospheric temperature, mimicking the steep temperature increase from the photosphere $(z=0)$ to the corona:

$$
T(z)=T_{\mathrm{ph}}+\frac{T_{\mathrm{cor}}-T_{\mathrm{ph}}}{2}\left(\tanh \frac{z-z_{\mathrm{cor}}}{w_{\mathrm{tr}}}+1\right)
$$

where $T_{\mathrm{ph}}=6109 \mathrm{~K}, T_{\text {cor }}=0.61 \mathrm{MK}, y_{\text {cor }}=2.12 \mathrm{Mm}$, and $w_{t r}=0.18 \mathrm{Mm}$. This profile creates an isothermal photosphericchromospheric layer at $0 \mathrm{Mm} \leqslant z<1.96 \mathrm{Mm}$, a transition region at $1.9 \mathrm{Mm} \leqslant z<2.3 \mathrm{Mm}$, and an isothermal corona at $2.3 \mathrm{Mm} \leqslant z<11.88 \mathrm{Mm}$. The atmospheric density is derived by solving the hydrostatic equation $d P / d z=-g z$, assuming a photospheric density of $\rho_{\mathrm{ph}}=1.67 \times 10^{-7} \mathrm{~g} \mathrm{~cm}^{-3}$. The atmospheric temperature (solid black) and density (solid blue) are shown in Figure 2. For comparison, we plot with dashed lines the temperature and density for the 1D model atmosphere (model C7) of Avrett \& Loeser (2008).

The initial magnetic field is the sum of two magnetic sources and a horizontal field:

$$
\boldsymbol{B}=\frac{F}{2 \pi} \frac{\hat{\boldsymbol{r}_{\boldsymbol{I}}}}{r_{1}^{2}}-\frac{F}{2 \pi} \frac{\hat{\boldsymbol{r}_{2}}}{r_{2}^{2}}-B_{0} \hat{\boldsymbol{x}}
$$

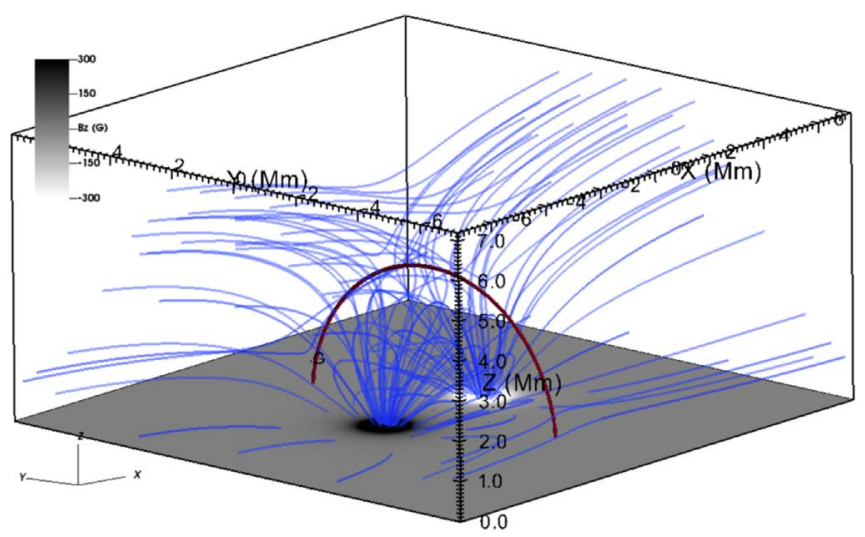

Figure 3. The 3D magnetic field configuration at $t=0$ (blue field lines) and the photospheric $B_{z}$ saturated at $\pm 300 G$. The red contour of $|B|<0.5 \mathrm{G}$ shows the location of a ring of null points.

Table 1

Initial Conditions for the Simulations

\begin{tabular}{lcc}
\hline \hline Name & $B_{0}(\mathrm{G})$ & $z_{s}(\mathrm{Mm})$ \\
\hline Case 1 & 15 & 3.6 \\
Case 2 & 30 & 2.6 \\
Case 3 & 47 & 2 \\
Case 4 & 53 & 1.8 \\
Case 5 & 114 & 0.9 \\
\hline
\end{tabular}

where

$$
\begin{aligned}
& \boldsymbol{r}_{1}=\left(x+d_{s}\right) \hat{\boldsymbol{x}}+y \hat{\boldsymbol{y}}+\left(z-z_{0}\right) \hat{\boldsymbol{z}}, \\
& \boldsymbol{r}_{2}=\left(x-d_{s}\right) \hat{\boldsymbol{x}}+y \hat{\boldsymbol{y}}+\left(z-z_{0}\right) \hat{\boldsymbol{z}},
\end{aligned}
$$

are the position vectors of the left and right sources, respectively, $d_{s}=1.8 \mathrm{Mm}$ is the distance of each source from the center, and $z_{0}=-0.36 \mathrm{Mm}$ is the depth of the sources below the photosphere (assumed outside the numerical domain). Each source has flux $F=2.1 \times 10^{19} \mathrm{Mx}$, and the resulting photospheric polarities have a maximum field strength of $2.6 \mathrm{kG}$. The size of the polarities at the photosphere, defined as the length across which $\left|B_{z}\right|>100 \mathrm{G}$, is $1.9 \mathrm{Mm}$ (Figure 4(a)). The photospheric flux of each photospheric polarity is $F_{m}=1.9 \times 10^{19} \mathrm{Mx}$. The horizontal field has a strength of $B_{0}=15 \mathrm{G}$ (case 1). The magnetic field configuration at $t=0$ is visualized in Figure 3. Other cases of $B_{0}$ were also examined, where $B_{0}$ was varied in order to vary the initial height of the null points in the atmosphere. The values of $B_{0}$ and the corresponding maximum height of the null point at $x=y=0$ at $t=0$ minutes are shown in Table 1 .

The boundary conditions are imposed in a similar way to Paper II. At the lower boundary (photosphere), the density and energy are assumed to have zero gradient. The simulation is driven by changing the magnetic field at the lower boundary using Equation (21) and varying the source positions $\pm d(t)$ from their initial values $\pm d_{s}$ according to $d(t)=d_{s}-x(t)$, where $x(t)$ is

$$
\begin{aligned}
x(t)= & v_{\max } \frac{w}{2}\left[\ln \left(\cosh \frac{t-t_{0}}{w}\right)-\ln \left(\cosh \frac{t_{0}}{w}\right)\right] \\
& +\frac{1}{2} v_{\max } t
\end{aligned}
$$


(a)

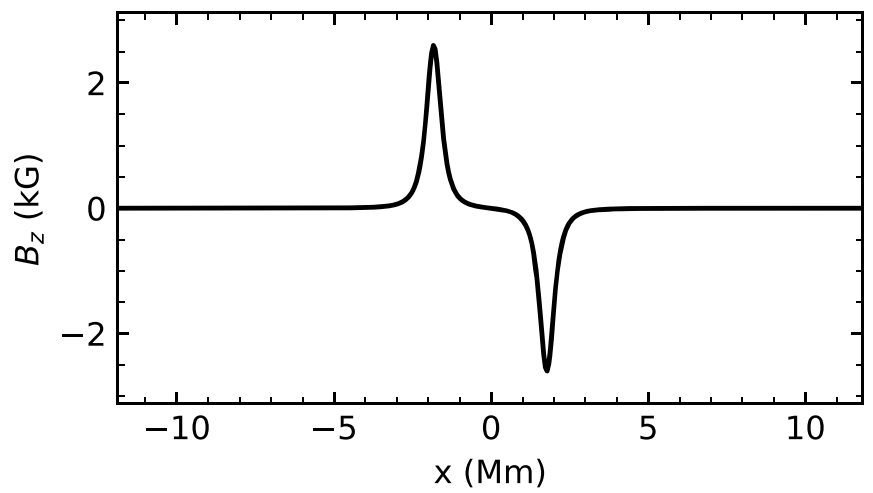

(b)

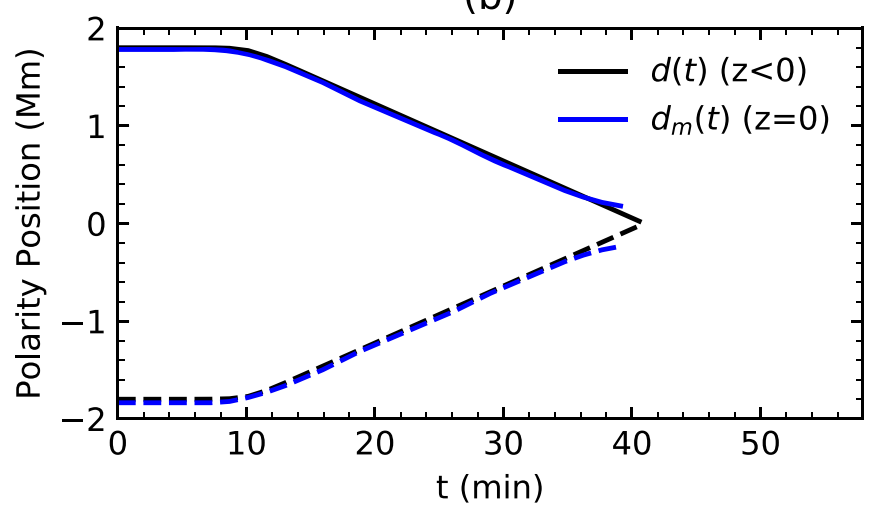

Figure 4. (a) Photospheric $B_{z}$ along the $x$-axis, at $y=0$. (b) The position of the sources ( $d$, black lines) and the position of the photospheric polarities $\left(d_{m}\right.$, blue lines) as a function of time.

and $v_{\max }=1 \mathrm{~km} \mathrm{~s}^{-1}, t_{0}=10.1$ minutes and $w=1.4$ minutes. This $x(t)$ leads to source velocities of $\pm v_{0}(t)$, where

$$
v_{0}(t)=\frac{1}{2} v_{\max }\left(\tanh \frac{t-t_{0}}{w}+1\right) .
$$

In Figure 4(b) (black line) we show the half-separation $(d(t))$ of the sources (below the photosphere, outside the numerical domain) as a function of time. The blue lines show the positions of the polarities at $z=0$ (found by measuring the location of maximum $B_{z}$ at the photosphere). The latter reflects the response of the photosphere to the driver. At the rest of the boundaries, we assume $\boldsymbol{v}=0$ and zero gradients for $\boldsymbol{B}, \rho, \epsilon$.

\subsection{Comparison of Theory with Simulation}

In this section, we discuss case 1 of Table 1 and use it to compare the simulation with the analytical theory summarized in Section 2.

\subsubsection{Brief Description of Simulation}

The 3D magnetic field at $t=0$ (Figure 3) shows that above the magnetic arcade, a semicircular ring of null points (red contour) extends from the photosphere and reaches a maximum height at the $x z$-midplane $(y=0)$. At that plane, the null is located in the corona at a height of $(x, z)=(0,3.6) \mathrm{Mm}$ (Figure 5(a)). To further visualize the ring of null points, in Figure 6 we show the plasma $\beta$ at the $y z$-midplane, and the positions of the null point according to Equation (6) (dashed line). Because the background atmosphere is stratified, the null (a)

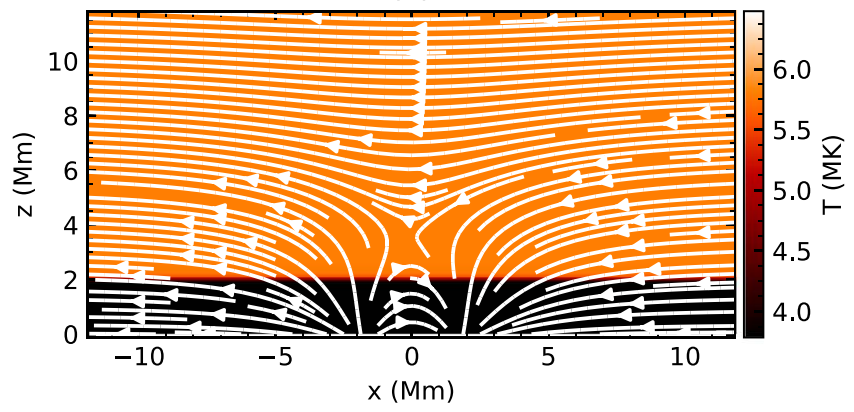

(b)

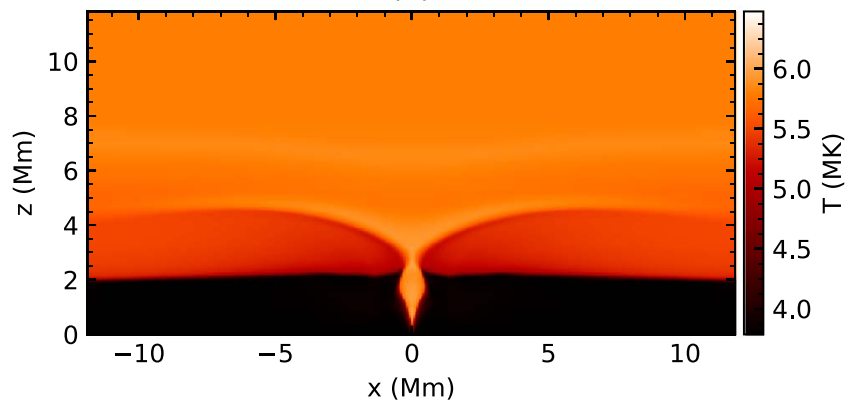

(c)

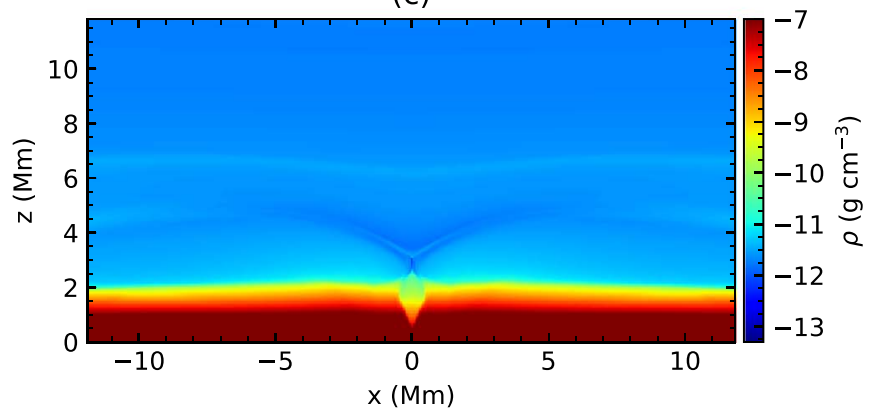

Figure 5. (a) Temperature and magnetic field at $t=0$ at the $x z$-midplane. (b) Temperature and (c) density at $t=43$ minutes at the $x z$-midplane.

points are located both inside regions of low plasma $\beta$ (between $y= \pm 3.5 \mathrm{Mm}$, red color around dashed line) and of high plasma $\beta$ (blue color around dashed line).

When the driver is switched on, reconnection is not driven across the whole semicircular ring of null points because of the different plasma $\beta$ environments. Instead, the converging photospheric polarities drive reconnection only between $y= \pm 3.5 \mathrm{Mm}$ (Figure 6(b)), which needs to be taken into account when comparing the theory with the simulation, and will be discussed later.

The energy released by reconnection heats locally the plasma, which spreads above and below the null points (and shows up as a "horizontal" heated region and an underlying heated arcade in Figure 5(b)), and is denser than the background atmosphere (Figure 5(c)).

After $t=36$ minutes, the photospheric polarities $\left(d_{m}(t)\right.$, blue lines, Figure 4(b)) do not keep following the driver (black lines). This is because the magnitude of the photospheric field has decreased to the point that $\beta>1$. As a result, the driver cannot move the overlying field anymore, therefore the reconnection at the null points gradually stops. Above the photosphere, there is still a remaining magnetic arcade. 
(a)

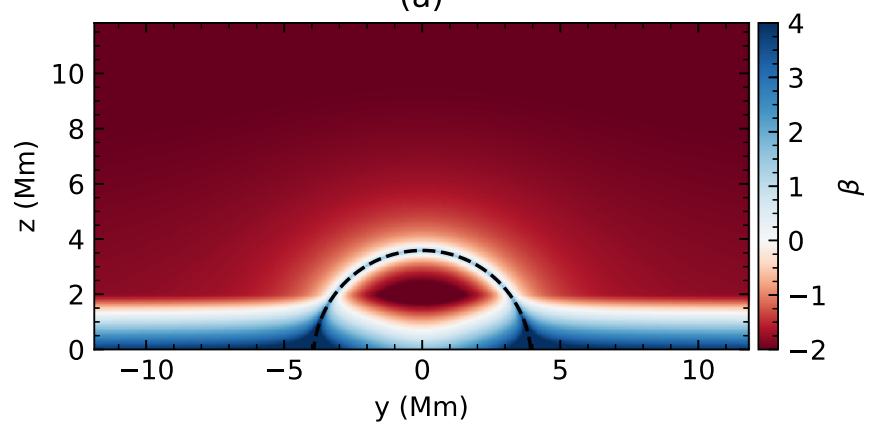

(b)

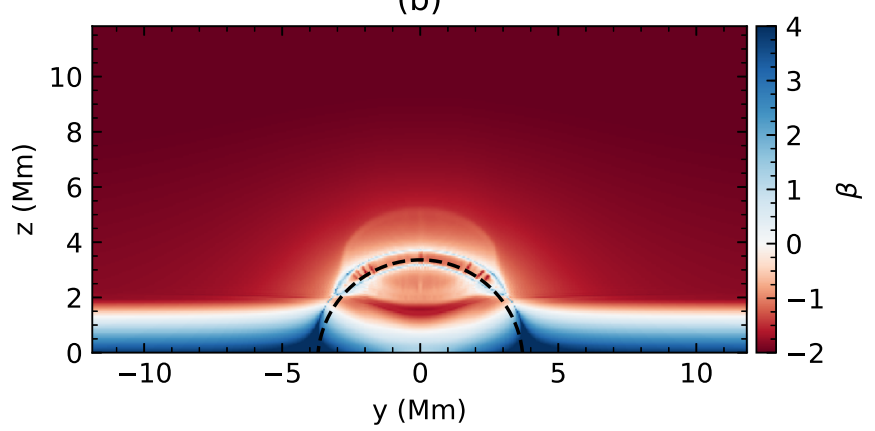

Figure 6. Plasma $\beta$ at the $y z$-midplane $(x=0)$ at (a) $t=0$ and (b) $t=17.4$ minutes. The dashed lines show the location of the null points according to Equation (6).

\subsubsection{Comparison Methodology}

When deriving the theory, we assumed that the converging polarities drive reconnection across the whole semicircular ring of null points (i.e., across the dashed line of Figure 6(a)). Therefore, we assumed that the length along which reconnection occurs is $\pi z_{S}$. In the simulation, however, reconnection is not driven across the whole semicircular ring because of the different plasma $\beta$ regions. Instead, the converging photospheric polarities drive reconnection only along a dashed line segment between $y= \pm 3.5 \mathrm{Mm}$ (Figure 6(b)). We call the length of that line segment $l$. To compare theory and simulation, the theory has to be adjusted to predict the energy released associated with the length $l$ rather than $\pi z_{S}$. To do so, we multiply Equations (12) and (13) by a correction factor of $l /\left(\pi z_{S}\right)$.

In Paper II, we compared the simulation with the theory using two approaches. The first was to make the theoretical estimates using the parameters characterizing the driver (e.g., the flux and time-distance profile of the sources below the photosphere). The second approach was to measure the photospheric and atmospheric response to the driver (e.g., measured photospheric flux and time-distance profile of the photospheric polarities) and use these with the theoretical expressions. It was found that the latter approach, which mimics an actual observation, gave a better comparison between simulation and theory, so that is the approach we adopt here.

To compare theory with simulation, we need to identify the characteristic surface around the current sheet as it evolves in time. The current sheet is a structure located approximately between $y= \pm 3.5 \mathrm{Mm}$ along the $y$-axis. At each point along the $y$-axis, we identify the vertical extent along the $z$-axis, tracing the arced shape of the current sheet (the distance between the between blue lines around $y= \pm 3.5 \mathrm{Mm}$, Figure 6(b)). To visualize this shape, in

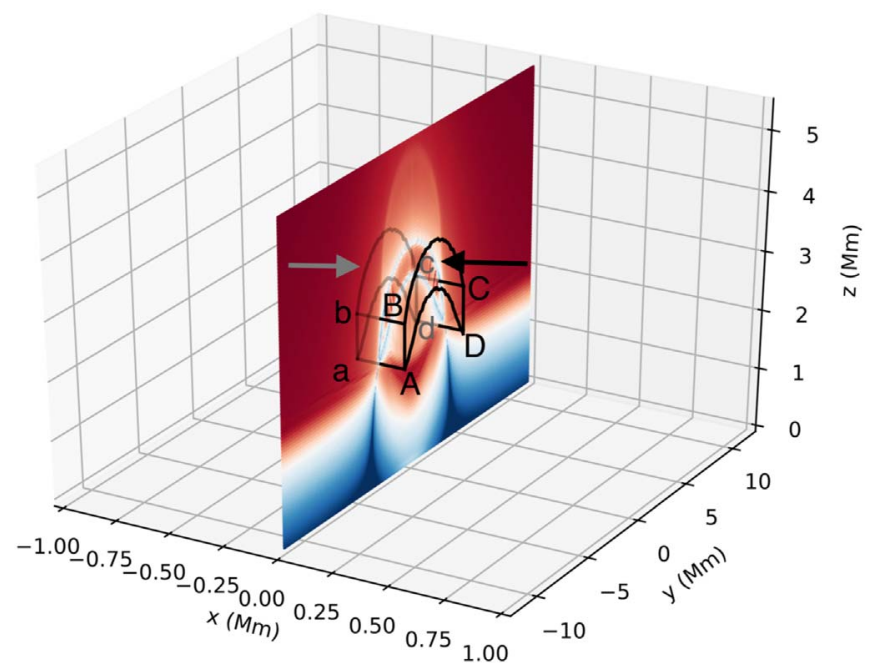

Figure 7. A $3 \mathrm{D}$ representation of the characteristic surface inside which the current sheet is located at $t=17.4$ minutes (solid lines). The red-blue plane is the same as that in Figure 6(b). The arrows show the sides through which plasma inflows into the current sheet.

Figure 7 we plot the $y z$-midplane of Figure 6(b) in a 3D volume. The traced arced black lines (e.g., AD and BC) follow the shape of the current sheet. We then identify the regions that are parallel to the current sheet and at a distance of $\Delta x=0.2 \mathrm{Mm}$ away from it (ABCD and abcd surfaces). This distance is such that the current density there is at least an order of magnitude lower than the one inside the current sheet. Using these two surfaces, we identify a three-dimensional surface, delineated by the black lines, inside which the current sheet is located. This shape is changing over time as the polarities converge.

We compare the theory with the simulation as follows. The length $(L)$ of the current sheet is taken for simplicity to be the vertical extent of the current sheet along the $z$-axis at $x=y=0$. We do so since the length of the current sheet is approximately radially symmetric (see Figure 6(b)). The values of the inflowing magnetic field strength $\left(B_{i}\right)$, inflowing velocity magnitude $\left(v_{i}\right)$, and inflowing density $\left(\rho_{i}\right)$ are taken as the average values at the surfaces parallel to the current sheet (ABCD and abcd, Figure 7). The total inflow of Poynting flux $\left(S_{i}\right)$ into the current sheet is measured by taking into account the sum of the Poynting flux through both the abcd and ABCD surfaces.

\subsubsection{Comparison of Theory and Simulation}

The inflow magnetic field strength $\left(B_{i}\right)$ and velocity magnitude $\left(v_{i}\right)$ are plotted in Figures 8(a) and (b). The solid lines are measured from the simulation and the dashed lines are the theoretical estimates using Equations (7) and 8, respectively. We see that the theory is in good agreement with the simulation.

To estimate the length of the current sheet using Equation (10) we have to measure $\alpha$ (Alfvén Mach number) and $v_{\mathrm{A} 0}$ using Equation (11). For $\alpha$, we use average values of the Alfvén Mach number between $t=10$ minutes and 40 minutes, during which the cancellation occurs, which is $\alpha=0.075$ (Figure 8(c)). This value of $\alpha$ is typical for fast reconnection (Priest 2014). Figure 8(d) shows the length of the current sheet from the simulation (solid line) and from the theoretical estimate (dashed line). Both are in good agreement. 
(a)

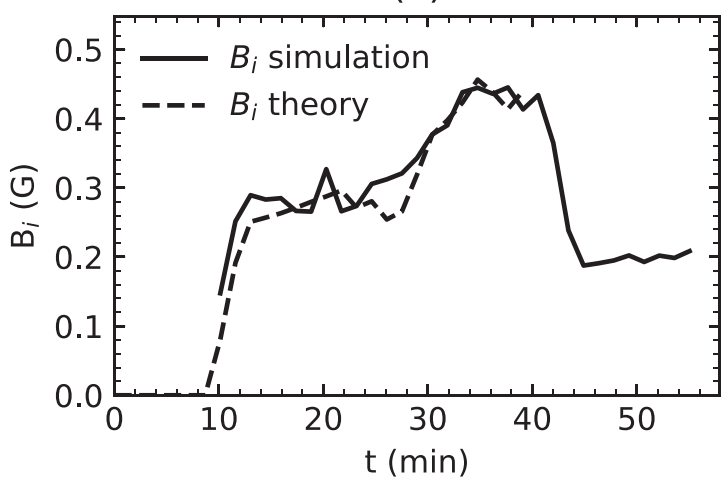

(c)

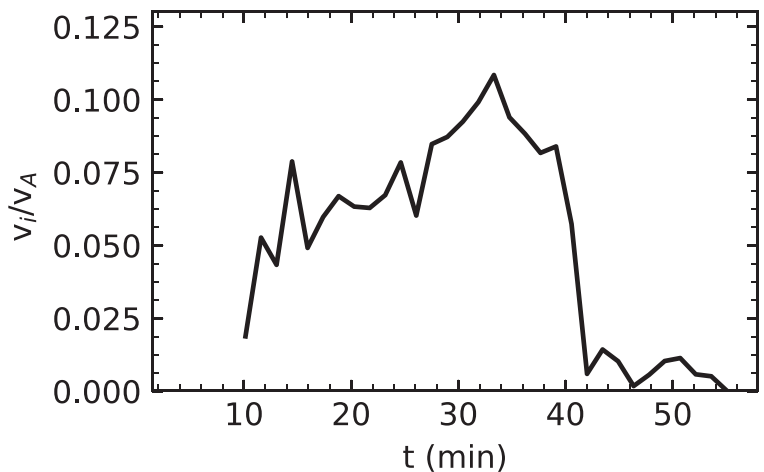

(b)

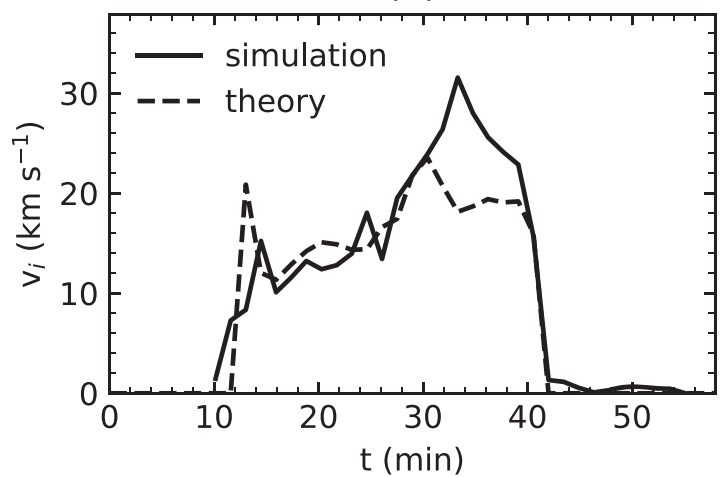

(d)

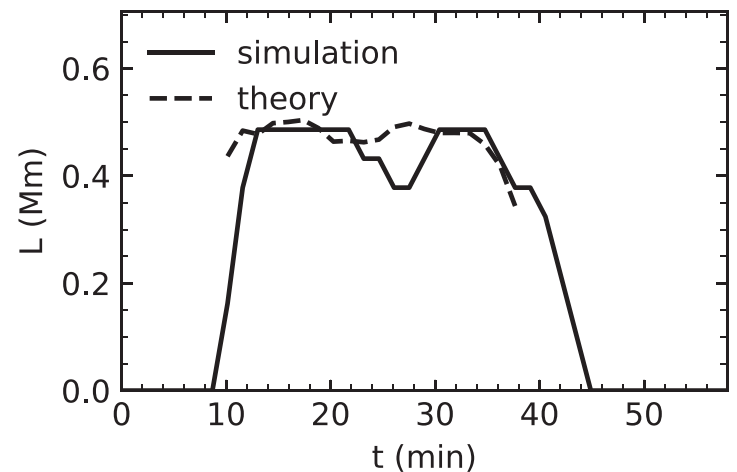

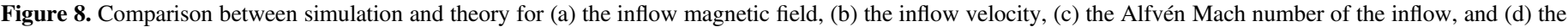
length of the current sheet.

We now estimate the Poynting flux into the current sheet. The measured Poynting inflow is shown in Figure 9 (solid line). The dashed line shows the analytical estimate using Equation (12). The analytical estimate becomes larger than the measured Poynting flux by up to a factor of 2 . This is because Equation (12) assumes that the length along which reconnection occurs is $\pi z_{s}$. However, as discussed previously, in the simulation, reconnection occurs only along a part of the semicircular ring of null points with length $l$. If we correct the theoretical prediction by taking this into account and multiply Equation (9) by a correction factor of $l /\left(\pi z_{S}\right)$, then the theory is in good agreement with the simulation (dotted-dashed line). Similar agreement is found for the other cases in Table 1.

In Paper II, we also compared the conversion of Poynting flux to kinetic and thermal energy. To do a similar analysis for the 3D simulation, we would have to calculate the energy integral term $-\int_{V} \eta \dot{j}^{2} d V$ and compare it with Equation (13). However, as discussed in Section 3, it is numerical resistivity that drives the reconnection at the current sheet. Therefore, the explicit value of $\eta$ is unknown, so we do not here compare the analytical expression for the release of heat with the simulation. We note, however, that the Poynting inflow is estimated well by theory, as are the parameters associated with the reconnection. So, we expect the expression for the conversion of Poynting flux inflow to heat to be also well estimated, since it is simply a proportion of the Poynting inflow.

\subsection{Atmospheric Response}

We now discuss the atmospheric response following reconnection. For this we vary the atmospheric field strength $B_{0}$ in order to change the initial maximum height of the nulls at

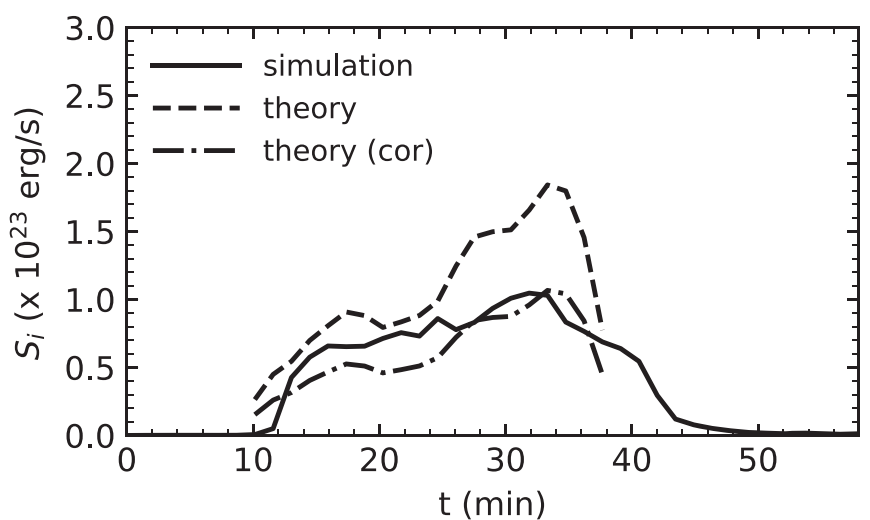

Figure 9. Comparison between the simulation, the theory and the "corrected" theory for the total Poynting influx.

$t=0$, thus initiating reconnection at different atmospheric heights. The initial maximum height of the nulls for different values of $B_{0}$ is shown in Table 1. These cases are similar to the ones used in Paper II, and are selected so that the initial maximum height of the nulls is placed either high in the corona (case 1), at base of the corona (case 2), at the middle of the transition region (case 3), at the base of transition region region (case 4), or near the photosphere (case 5). These heights are shown against the initial stratification in Figure 2 (as vertical lines).

In Paper II we found that, during the convergence of the polarities, starting at its maximum height, the current sheet moved toward the photosphere, thus the reconnection occurred at progressively lower atmospheric heights. When the reconnection 

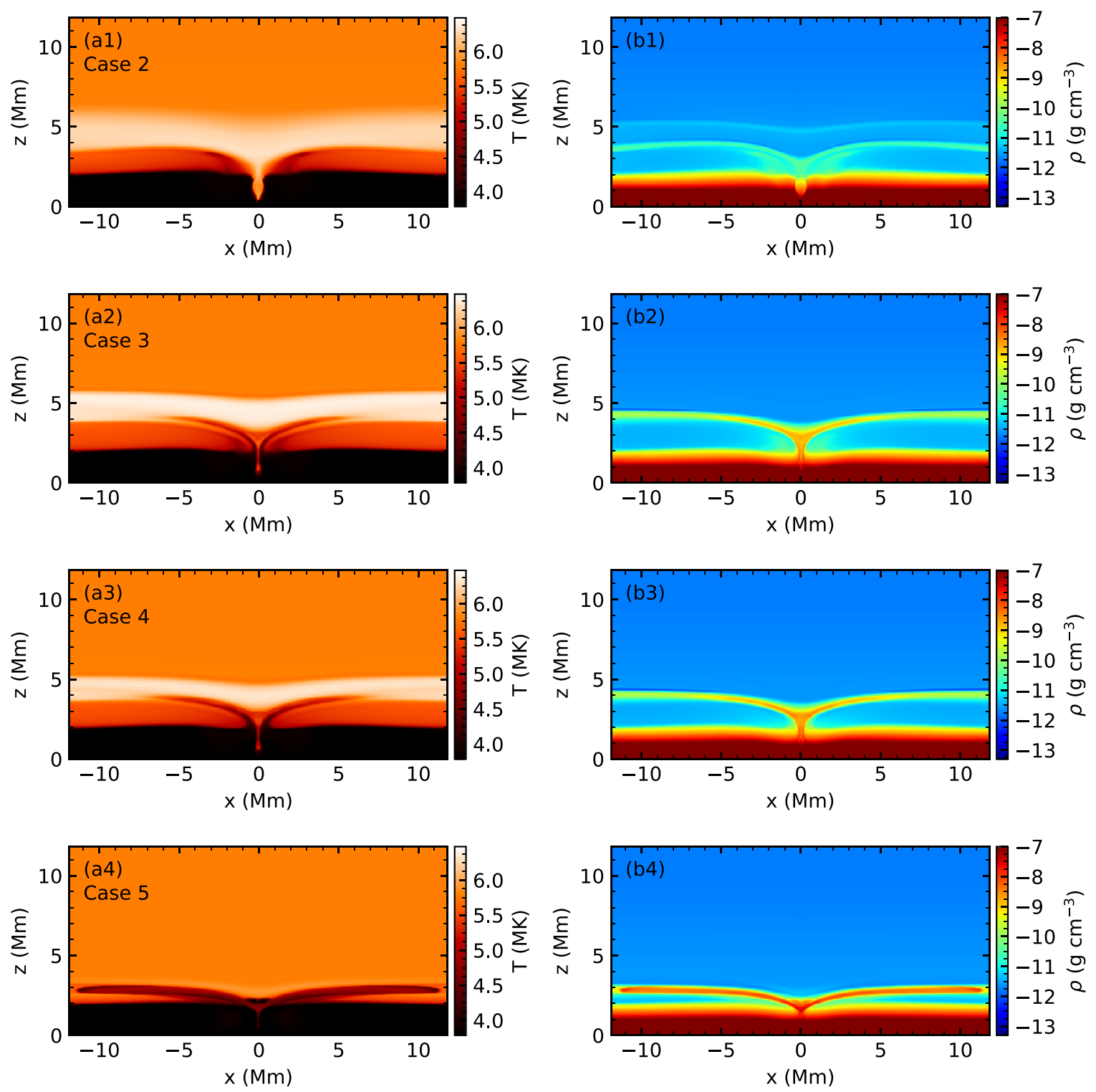

Figure 10. Temperature (left column) and density (right column) for cases 2-5 at $y=0$ for $t=43$ minutes.

occurred in the corona or upper transition region, the resulting outflow was hot. In contrast, when the reconnection occurred at lower heights, a cool outflow was also produced. Therefore, depending on the height of the null point, either hot ejections or cool ones, or a combination of both hot and cool ejections were formed. These hot and cool outflows were formed with or without a time difference, depending on the initial conditions. The same qualitative behavior is found in the $3 \mathrm{D}$ simulations, with the addition of spatial effects due to the 3D extent of the current sheet.

Figure 10 shows the temperature and density for cases 2 to 5 at the $y=0$ midplane after the driving has stopped. (Case 1 is shown in Figure 5.) Depending on the height of the null, the resulting outflows can be either hot or cool or can consist of a combination of both hot and cool plasmas. By examining the time evolution of the maximum velocity of the hot $(T>1 \mathrm{MK})$ and cool $(T<0.2 \mathrm{MK})$ outflows at that plane (Figure 11), we find that, when the null point is initially located higher in the atmosphere, the hot ejection is produced first ( $t=12$ minutes), and the cool ejection is produced later ( $t=30$ minutes) (panels (a)). For a null initially located lower in the atmosphere, the cool and the hot ejections have less temporal separation (cool ejections appear from $t=16$ minutes and $t=12$ minutes, (b) and (c)). For a null close to the photosphere, only a cool ejection is formed (d). These results are qualitatively similar to those of the 2D simulations in Paper II.

However, in 3D, instead of having only one null point at one height, we have a ring of nulls (or, more generally, a separator), so the convergence of the polarities drives reconnection simultaneously at many different heights (e.g., Figure 6(b)). Therefore, reconnection can occur at different heights not only because the current sheet moves downward during the convergence of the polarities, but also because of the spread of nulls or a separator over a range of heights. This produces both hot and cool ejections with a spatial offset.

To visualize the above, in Figure 12 we show for case 3 the temperature at an offset plane $(y=-3.5 \mathrm{Mm})$ and at the midplane of the numerical domain $(y=0 \mathrm{Mm})$, at three different times ( $t=19$ minutes, first row; $t=29$ minutes, second row; $t=43$ minutes, third row). At the $y=-3.5 \mathrm{Mm}$ plane, the null at $t=0$ is located lower in the atmosphere in 
(a)

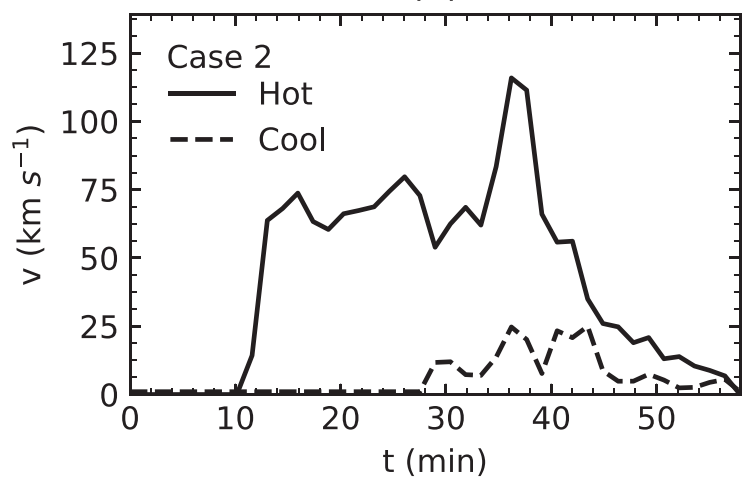

(c)

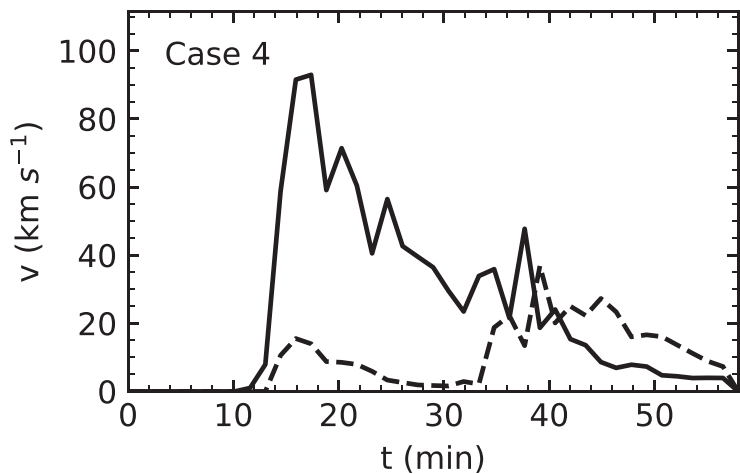

(b)

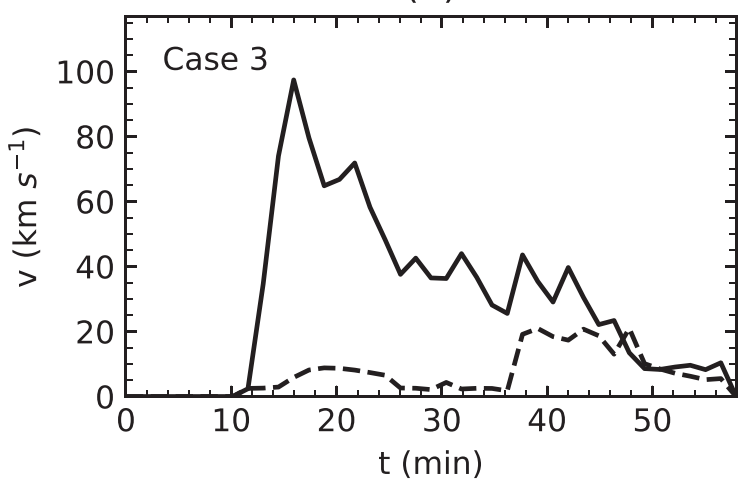

(d)

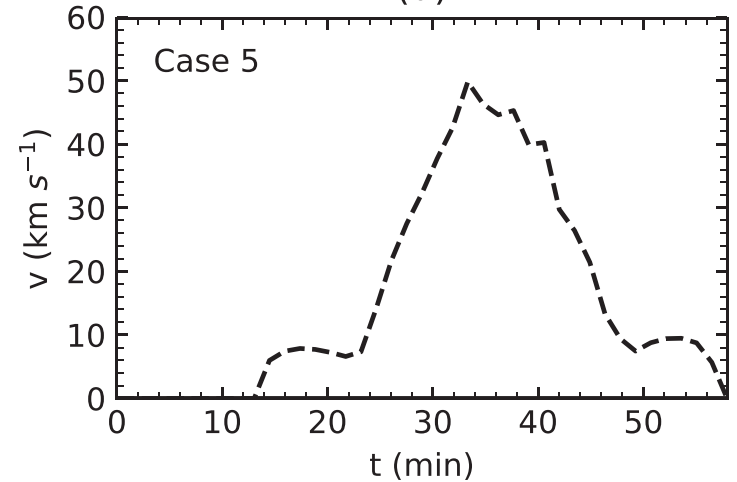

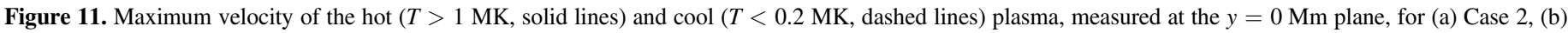
Case 3, (c) Case 4, and (d) Case 5.

comparison to the null at the $y=0 \mathrm{Mm}$ plane. Therefore, at that plane the convergence of the polarities results in mostly cool ejections (panels (a1)-(a3)). At the same time, at the $y=0$ plane, the ejections are initially hot (panels (b1) and (b2)), while cool ejections form with a time delay as the null progressively moves lower (panel (b3)). The latter cool outflows are spatially offset in comparison to the $y=-3.5 \mathrm{Mm}$ ones and develop at different times. In addition, panels (a1) and (b1) show that during the initial stages of the energy release, the hot ejection at the $y=0 \mathrm{Mm}$ plane is formed sooner than the offset cool one at the $y=-3.5 \mathrm{Mm}$ plane. This is because more energy is needed to accelerate upward the denser material located lower in the atmosphere.

We further demonstrate these spatial effects by computing 2D maps of the temporal evolution of the maximum velocity of the hot $(T>1 \mathrm{MK})$ and cool $(T<0.2 \mathrm{MK})$ outflows at all $y$ planes (Figure 13). In Case 1 we do not find any cool component, as the reconnection occurs mainly in the corona. In Case 2, we find the first signs of spatially offset hot and cool flows. At $t=14$ minutes, the hot outflow originates from around $y=0$, whereas the two offset cool outflows originate from around $y \approx \pm 3.5 \mathrm{Mm}$. Throughout the cancellation, these cool outflows are at different spatial locations from the hot outflow. At $t>30$ minutes another cool outflow starts to appear around $y=0$, since the null point at that plane has now moved lower. This flow is cospatial with the hot outflow originating from a similar location. A gap appears between the cool outflows in panel (a2) around $t=30-45$ minutes. Asymmetric shrinking of the ring of null due to differences in $\beta$ causes a pressure gradient below the ring of nulls, which lifts the dense atmosphere around $y=0$ upward. Therefore, at $y=0$, the dense atmosphere is higher than that at $y \approx 2$, so reconnection at $y=0$ produces a cooler outflow than at $y \approx 2$, creating the gap. In cases 3 and 4, the cool outflows are again spatially offset, with their magnitudes becoming stronger as the nulls are located lower in the atmosphere. In addition, the cool outflows around $y=0$ start to appear earlier, as in Paper II. In Case 5, we find a cool outflow originating only from the central part of the canceling region.

Adding to our findings from Paper II, our results indicate that, depending on the height of the null point and the parameters of the cancellation, during flux cancellation: (i) hot ejections or cool ejections or a combination of both hot and cool ejections can be formed, (ii) these can be formed with or without a time difference and (iii) these can be formed with or without a spatial offset.

\section{Discussion}

In Paper I we presented a theoretical model on how magnetic reconnection driven by photospheric flux cancellation can act as a mechanism for energizing coronal loops and heating the chromosphere. In Paper II we numerically validated our theoretical estimates by the means of 2D simulations of two converging polarities inside a stratified atmosphere with a horizontal background field. In the present work, we further the numerical validation and study the atmospheric response to the cancellation by performing 3D simulation.

In our theoretical model we assumed that as the two polarities converge, reconnection is driven at a circular ring of null points that extends from the photosphere up to a maximum height in the atmosphere. In the simulations, because the field is inside a stratified atmosphere, the convergence of the field 

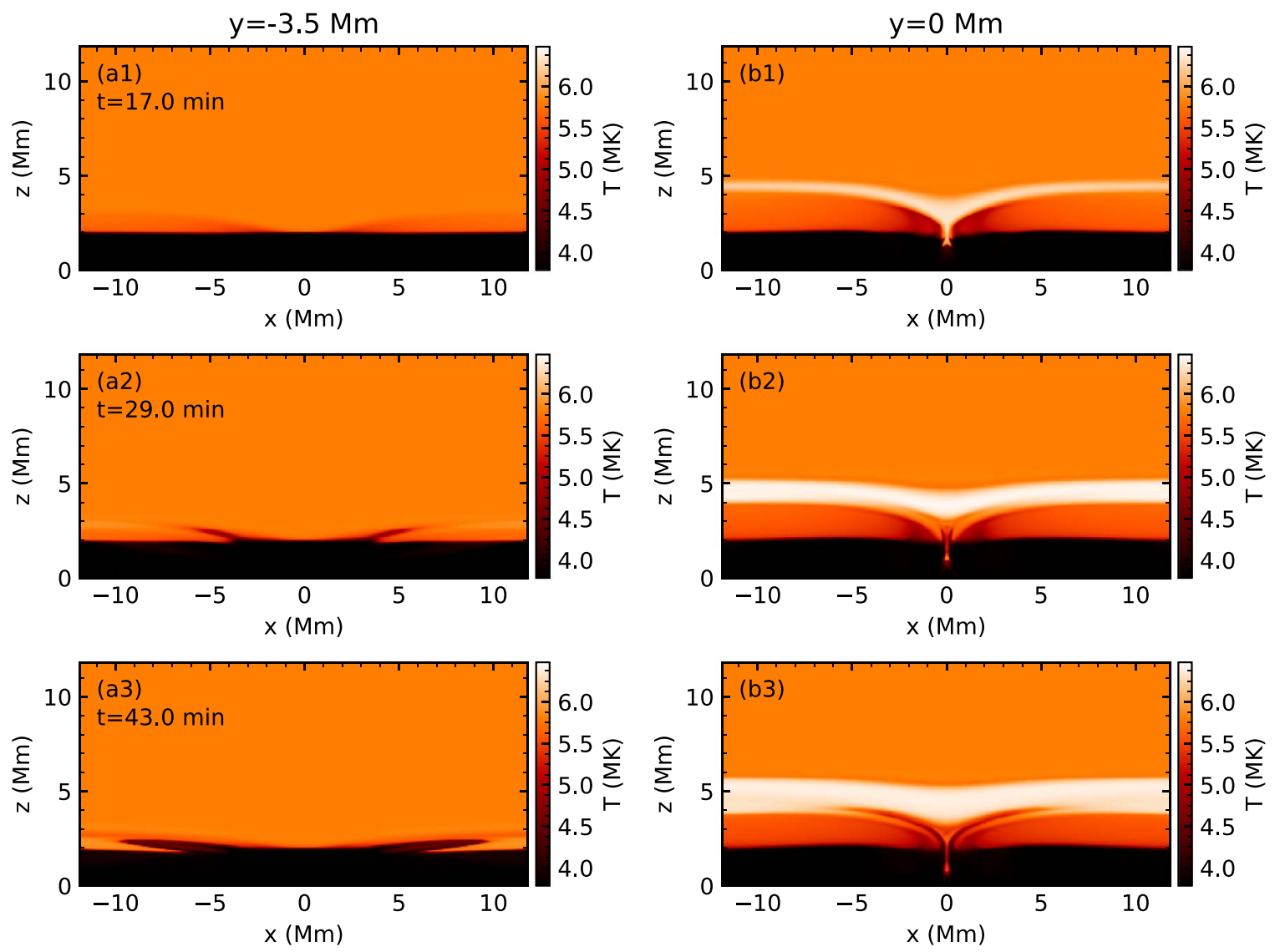

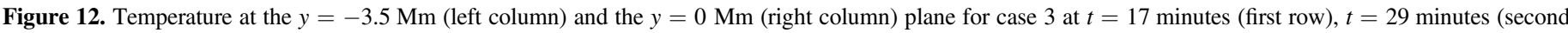
row) and $t=43$ minutes (third row).

does not drive reconnection along the whole length of that circular ring. It does so, only along the length of the ring of nulls that is located in a low $\beta$ environment. The part of the ring of nulls located in a high $\beta$ environment does not react to the convergence of the polarities. When comparing the theoretical model to the 3D simulation, we found that the simulated total Poynting flux flowing into the current sheet differed from the theoretical estimate by a factor of $\sim 2$. After correcting the theoretical estimate to account for the fact that only a part of the ring of nulls is involved in the reconnection, the theory was in excellent agreement with the simulation.

In Paper II, besides the Poynting influx, we compared the energy release rate from the simulation with the theory. We do not make such a comparison in the current paper, since the numerical resistivity dominates. However, all the quantities involved in the analytical estimation of the rate of energy release (inflowing magnetic field strength and velocity, current sheet length, Poynting influx) are in agreement with the simulation. Therefore, it is expected that the analytical estimate of the rate of energy release would also be in agreement with a simulation with much higher resolution. Therefore, the energy released during photospheric cancellation can be estimated accurately with knowledge of the parameters of the cancellation.

We also studied the atmospheric response to the reconnection and found that both cool and hot ejections may be generated. In this work we have mainly focused on how the formation of the different ejections depends on the height of the energy release. Furthermore, we comment briefly on the acceleration of the ejections, without going into too much detail as this is beyond the scope of the current paper. In the vicinity of the reconnection region, shocks accelerate the reconnection outflows. These outflows collide with the overlying horizontal ambient field which diverts them sideways. There, further shocks may develop due to an increase in the local compression. The diverted flows are the main ejections discussed in this paper.

In addition, the collision of the reconnection outflow with the overlying horizontal ambient field accelerates the plasma of the overlying field. This process is more important when the reconnection occurs lower in the atmosphere, as it accelerates denser material, forming another cool ejection. When the reconnection occurs lower in the atmosphere both the diverted reconnection outflow and the displaced dense material can be cool, but the density and temperature of the two can be quite different. Similar results were obtained in the 2D simulations of Part II. The acceleration of the ejections is similar to what is found in $2.5 \mathrm{D}$ numerical simulations of reconnection at different atmospheric heights driven by flux emergence (Takasao et al. 2013).

The temporal evolution and plasma properties of the jets were also examined, validating our previous results and extending them to include 3D spatial effects. In summary, our results indicate that, depending on the properties of the canceling region: (i) either hot ejections or cool ones or a combination of both hot and cool ejections can be formed, (ii) these can be formed with or without a time difference and (iii) with or without a spatial offset. The spatial offset is simply due 

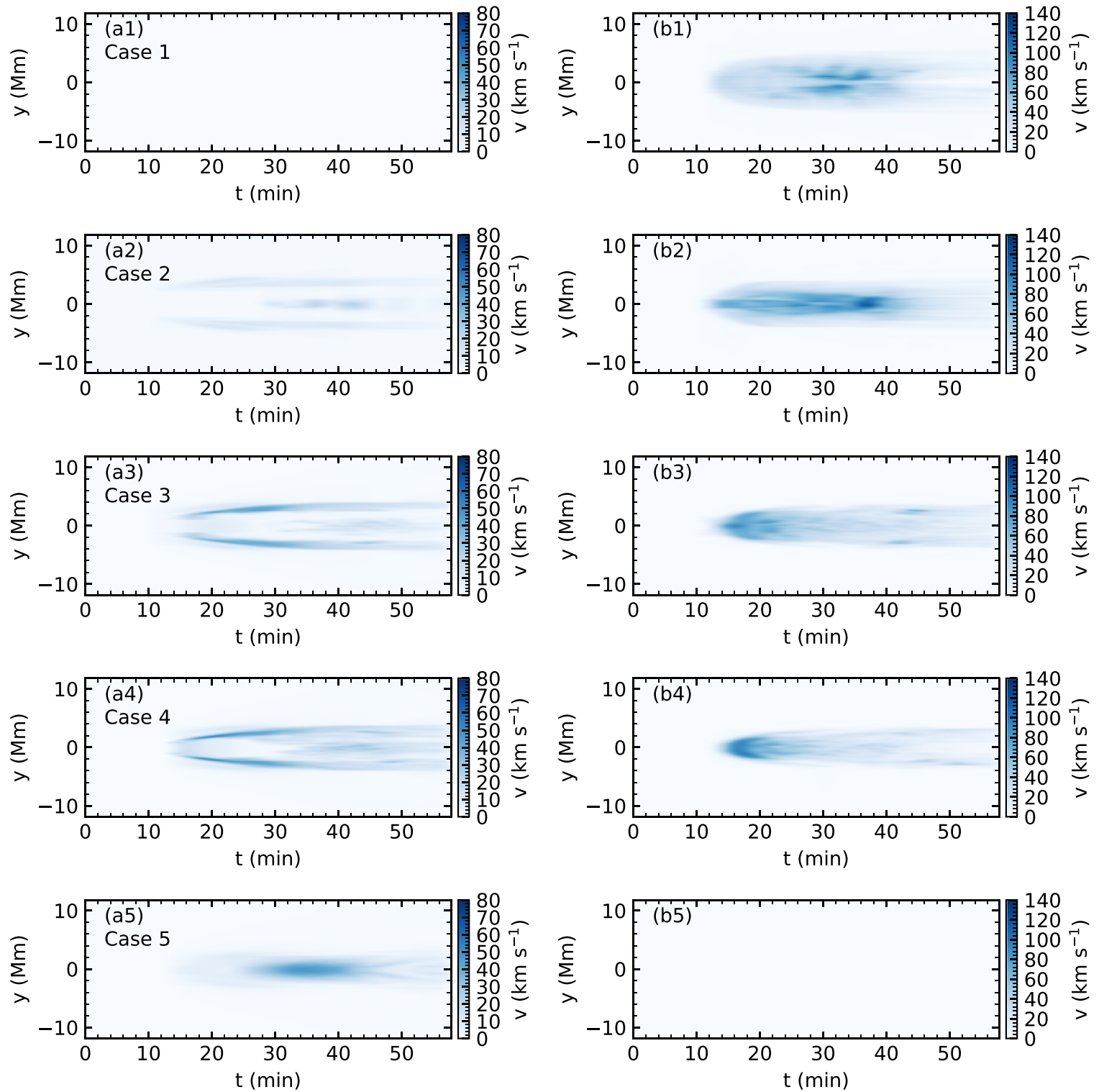

Figure 13. 2D maps of the time evolution of the maximum velocity at each $y$-plane for the cool $(T<0.2 \mathrm{MK}$, left column) and the hot $(T>1 \mathrm{MK}$, right column) plasma components, for all the different cases (rows).

to the fact that the nulls along the ring are located at different heights. The time difference can occur both due to reconnection occurring at different spatial locations, where a different amount of energy is needed to energize plasma of different densities, leading to a time difference between the ejections, and due to a current sheet moving lower in the atmosphere later in the cancellation. In addition, note that the current sheet has a vertical length, and therefore reconnection occurs at multiple heights at any given time along the vertical extend of it. We note that different kinds of ejection can be formed (e.g., either hot or cool or both) when the reconnection at different atmospheric heights is driven by flux emergence (e.g., Shibata 1999).

The hot ejections in our numerical investigation have temperatures of $0.6-2 \mathrm{MK}$, densities of $10^{-13}-10^{-15} \mathrm{~g} \mathrm{~cm}^{-3}$, velocities of $25-125 \mathrm{~km} \mathrm{~s}^{-1}$, and widths $4-8 \mathrm{Mm}$. The hot ejections always reach the boundary of the domain, so their lengths are at least $12 \mathrm{Mm}$. Such values are typical for smallscale coronal and transition region jets. The cool ejections have temperatures of $0.01-0.2 \mathrm{MK}$, densities of $10^{-12}-10^{-14} \mathrm{~g} \mathrm{~cm}^{-3}$, velocities of $5-50 \mathrm{~km} \mathrm{~s}^{-1}$, widths of $2-4 \mathrm{Mm}$, and lengths of $3-10 \mathrm{Mm}$. These are typical values for surges. In addition, in case 5 we find that an even cooler ejection of photospheric or chromospheric temperature (around $6300 \mathrm{~K}$ ) is produced, as described in the previous paragraph. In some of the cases, ejections of intermediate temperatures of $0.2-0.6 \mathrm{MK}$ are found as the null progressively moves toward the photosphere. The above values are similar to the ones reported for hot and cool ejections associated with surges and hot jets driven by reconnection during flux emergence (see e.g., Moreno-Insertis \& Galsgaard 2013; MacTaggart et al. 2015; Nóbrega-Siverio et al. 2016). We expect that plasmoid-induced reconnection (e.g., Peter et al. 2019) can affect the temperature and density of the jets, but our main conclusion about the time differences and spatial offsets of the outflows should not be affected. The physical size and properties of these jets could also be affected by the addition of an oblique ambient field and by changing the properties of the canceling polarities. For example, the width of 
ejections will depend on the extent of the current sheet along which reconnection occurs, which will depend on the sizes and fluxes of the photospheric polarities, in addition to the ambient field strength and direction.

Note that in cases where the photospheric polarities are driven for a smaller time, mimicking a shorter-lasting flux cancellation, the null point would reconnect for a shorter time. Therefore, depending on the properties of the reconnection region, these outflows could be shorter-lasting and associated with a shorter burst of energy release. In principle, depending on the specifics of the canceling region (e.g., the local plasma $\beta$ distribution, the photospheric fluxes, the sizes of the polarities, the convergence speed, the flux cancellation rate, ambient magnetic field strength and orientation, etc.), various combinations of the manifestations of the ejections found in our simulations could occur.

So our results demonstrate that, in canceling regions, a wide spectrum of ejections can be produced, with a wide range of different temperatures and sizes associated with Ellerman bombs, UV bursts, and IRIS bombs and other hot and cool ejections (Yang et al. 2013; Vissers et al. 2015; Reid et al. 2016; Rutten 2016; Nelson et al. 2017; van der Voort et al. 2017; Tian et al. 2018; Young et al. 2018; Chen et al. 2019; Guglielmino et al. 2019; Huang et al. 2019; Madjarska 2019; Tiwari et al. 2019; Ortiz et al. 2020). The predicted spatial offsets and time delays for hot and cool ejections are in accordance with observational findings as, for example, surges and EUV/X-ray jets with time delays of $\sim 5-10$ minutes have been recorded (Schmieder et al. 1994; Alexander \& Fletcher 1999; Chae et al. 1999; Jiang et al. 2007). Also, hot and cool ejections can appear to be cotemporal and be either spatially offset or cospatial (e.g., Mulay et al. 2017; Kontogiannis et al. 2020), or only cool ejections can form (e.g., surges, Ortiz et al. 2020). We note further that similar energetic events at the feet of coronal loops could possibly create jet-like structures observed at these locations (Chitta et al. 2017b, 2017a). In addition, given the increasing evidence for the presence of unresolved minority polarities in the photosphere (e.g., Smitha et al. 2017; Solanki et al. 2017), such ejections resulting from flux cancellation can give rise to jetlike features found inside plages and strong networks associated with the unresolved underlying minority polarities (Wang 2016; Chitta et al. 2019; Wang et al. 2019). We expect that other small-scale phenomena, such as spicules, could be associated with reconnection at lower atmospheric heights between converging canceling polarities, and can potentially be associated with the cool outflows predicted from our model for different values of parameter space (e.g., Samanta et al. 2019).

Our results show that the local plasma $\beta$ is important for the production of outflows and energy bursts. Because reconnection is driven along the part of the ring of nulls located in a low$\beta$ plasma region, only that region will produce jets, thus the spatial extent of the jets will depend on the local plasma $\beta$. A detailed assessment of the role of the local plasma $\beta$ on the spatial extent of the jets produced by a canceling region would require a different modeling approach than the one employed here. We have imposed an initial potential field in a simple stratified atmosphere, for which the plasma density and temperature in the magnetized region simply follow the stratification. However, self-consistent flux emergence simulations show that the plasma density and temperature in an emerged field can be very different from the background stratification, since the adiabatic expansion of the field naturally forms cooler "bubbles" of magnetized plasma (e.g., Archontis et al. 2004; Leake \& Linton 2013; Nóbrega-Siverio et al. 2020). During our numerical experiment, we have not incorporated such an effect. Simulations where the polarities emerge self-consistently, interact across an extended vertical current sheet, and produce transient jet-like events (e.g., Archontis et al. 2013; Syntelis et al. 2015), Ellerman bombs (e.g., Archontis \& Hood 2009; Danilovic et al. 2017), UV bursts, and the spectral imprints of the resulting hot and cool outflows (Hansteen et al. 2017, 2019), are better suited for a detailed study of the role of plasma $\beta$ on the spatial extent of the jets.

In this work, we have further validated our analytical theory using 3D numerical computations, providing support to our suggestion that nanoflares driven by magnetic flux cancellation can be an important mechanism for chromospheric and coronal heating, as proposed in Paper I, building upon recent observational findings. Furthermore, our models suggest that a plethora of different hot and cool outflows produced with/ without time difference and spatial offset in small-scale canceling regions, represent a wide range of different manifestations of cancellation-driven reconnection. In future, we aim to extend our model by including different orientations of the horizontal field, including oblique fields, and examining different magnetic configurations.

This research has made use of NASA's Astrophysics Data System. This work was supported by computational time granted from the Greek Research and Technology Network (GRNET) in the National HPC facility ARIS. P.S. acknowledges support by the ERC synergy grant "The Whole Sun."

\section{ORCID iDs}

P. Syntelis (1) https://orcid.org/0000-0002-6377-0243

E. R. Priest (iD https://orcid.org/0000-0003-3621-6690

\section{References}

Alexander, D., \& Fletcher, L. 1999, SoPh, 190, 167

Arber, T., Longbottom, A., Gerrard, C., \& Milne, A. 2001, JCoPh, 171, 151

Archontis, V., \& Hood, A. W. 2009, A\&A, 508, 1469

Archontis, V., Hood, A. W., \& Tsinganos, K. 2013, ApJ, 778, 42

Archontis, V., Moreno-Insertis, F., Galsgaard, K., Hood, A., \& O'Shea, E. 2004, A\&A, 426, 1047

Avrett, E. H., \& Loeser, R. 2008, ApJS, 175, 229

Chae, J., Qiu, J., Wang, H., \& Goode, P. R. 1999, ApJL, 513, L75

Chen, Y., Tian, H., Peter, H., et al. 2019, ApJL, 875, L30

Chitta, L. P., Peter, H., \& Solanki, S. K. 2018, A\&A, 615, L9

Chitta, L. P., Peter, H., Solanki, S. K., et al. 2017a, ApJS, 229, 4

Chitta, L. P., Peter, H., Young, P. R., \& Huang, Y.-M. 2017b, A\&A, 605, A49

Chitta, L. P., Sukarmadji, A. R. C., Rouppe van der Voort, L., \& Peter, H. 2019, A\&A, 623, A176

Danilovic, S., Solanki, S. K., Barthol, P., et al. 2017, ApJS, 229, 5

Guglielmino, S. L., Young, P. R., \& Zuccarello, F. 2019, ApJ, 871, 82

Hansteen, V., Ortiz, A., Archontis, V., et al. 2019, A\&A, 626, A33

Hansteen, V. H., Archontis, V., Pereira, T. M. D., et al. 2017, ApJ, 839, 22

Hong, J., Ding, M. D., \& Cao, W. 2017, ApJ, 838, 101

Huang, Z., Li, B., \& Xia, L. 2019, STP, 5, 58

Huang, Z., Mou, C., Fu, H., et al. 2018, ApJL, 853, L26

Jiang, Y. C., Chen, H. D., Li, K. J., Shen, Y. D., \& Yang, L. H. 2007, A\&A, 469, 331

Kim, Y.-H., Yurchyshyn, V., Bong, S.-C., et al. 2015, ApJ, 810, 38

Klimchuk, J. A. 2006, SoPh, 234, 41

Kontogiannis, I., Tsiropoula, G., Tziotziou, K., et al. 2020, A\&A, 633, A67

Leake, J. E., \& Linton, M. G. 2013, ApJ, 764, 54 
Libbrecht, T., Joshi, J., Rodríguez, J. D. L. C., Leenaarts, J., \& Ramos, A. A. 2017, A\&A, 598, A33

Longcope, D. W. 1998, ApJ, 507, 433

MacTaggart, D., Guglielmino, S. L., Haynes, A. L., Simitev, R., \& Zuccarello, F. 2015, A\&A, 576, A4

Madjarska, M. S. 2019, LRSP, 16, 2

Meyer, C. D., Balsara, D. S., \& Aslam, T. D. 2012, MNRAS, 422, 2102

Moreno-Insertis, F., \& Galsgaard, K. 2013, ApJ, 771, 20

Mulay, S. M., Del Zanna, G., \& Mason, H. 2017, A\&A, 606, A4

Nelson, C. J., Doyle, J. G., \& Erdélyi, R. 2016, MNRAS, 463, 2190

Nelson, C. J., Freij, N., Reid, A., et al. 2017, ApJ, 845, 16

Nóbrega-Siverio, D., Moreno-Insertis, F., \& Martínez-Sykora, J. 2016, ApJ, 822,18

Nóbrega-Siverio, D., Moreno-Insertis, F., Martínez-Sykora, J., Carlsson, M., \& Szydlarski, M. 2020, A\&A, 633, A66

Ortiz, A., Hansteen, V. H., Nóbrega-Siverio, D., \& van der Voort, L. R. 2020, A\&A, 633, A58

Parker, E. N. 1979, Cosmical Magnetic Fields. Their Origin and their Activity (Oxford: Clarendon)

Parker, E. N. 1988, ApJ, 330, 474

Parnell, C. E., \& De Moortel, I. 2012, RSPTA, 370, 3217

Parnell, C. E., \& Priest, E. R. 1995, GApFD, 80, 255

Peter, H., Huang, Y. M., Chitta, L. P., \& Young, P. R. 2019, A\&A, 628, A8

Peter, H., Tian, H., Curdt, W., et al. 2014, Sci, 346, 1255726

Priest, E. 2014, Magnetohydrodynamics of the Sun (Cambridge: Cambridge Univ. Press)

Priest, E. R., Chitta, L. P., \& Syntelis, P. 2018, ApJL, 862, L24

Priest, E. R., Heyvaerts, J. F., \& Title, A. M. 2002, ApJ, 576, 533

Priest, E. R., Parnell, C. E., \& Martin, S. F. 1994, ApJ, 427, 459

Reid, A., Mathioudakis, M., Doyle, J. G., et al. 2016, ApJ, 823, 110

Rezaei, R., \& Beck, C. 2015, A\&A, 582, A104

Rutten, R. J. 2016, A\&A, 590, A124

Rutten, R. J., van der Voort, L. H. M. R., \& Vissers, G. J. M. 2015, ApJ, 808, 133
Şahin, S., Yurchyshyn, V., Kumar, P., et al. 2019, ApJ, 873, 75

Samanta, T., Tian, H., Yurchyshyn, V., et al. 2019, Sci, 366, 890

Schmieder, B., Golub, L., \& Antiochos, S. K. 1994, ApJ, 425, 326

Shibata, K. 1999, Ap\&SS, 264, 129

Smitha, H. N., Anusha, L. S., Solanki, S. K., \& Riethmüller, T. L. 2017, ApJS, 229, 17

Solanki, S. K., Barthol, P., Danilovic, S., et al. 2010, ApJL, 723, L127

Solanki, S. K., Riethmüller, T. L., Barthol, P., et al. 2017, ApJS, 229, 2

Syntelis, P., Archontis, V., Gontikakis, C., \& Tsinganos, K. 2015, A\&A, 584, A10

Syntelis, P., Priest, E. R., \& Chitta, L. P. 2019, ApJ, 872, 32

Takasao, S., Isobe, H., \& Shibata, K. 2013, PASJ, 65, 62

Tian, H., Xu, Z., He, J., \& Madsen, C. 2016, ApJ, 824, 96

Tian, H., Zhu, X., Peter, H., et al. 2018, ApJ, 854, 174

Tiwari, S. K., Alexander, C. E., Winebarger, A. R., \& Moore, R. L. 2014, ApJL, 795, L24

Tiwari, S. K., Panesar, N. K., Moore, R. L., et al. 2019, ApJ, 887, 56

Toriumi, S., Katsukawa, Y., \& Cheung, M. C. M. 2017, ApJ, 836, 63

Ulyanov, A. S., Bogachev, S. A., Loboda, I. P., Reva, A. A., \& Kirichenko, A. S. 2019, SoPh, 294, 128

van Ballegooijen, A. A., \& Martens, P. C. H. 1989, ApJ, 343, 971

van der Voort, L. R., De Pontieu, B., Scharmer, G. B., et al. 2017, ApJL, 851, L6

Vissers, G. J. M., Rouppe van der Voort, L. H. M., Rutten, R. J., Carlsson, M., \& De Pontieu, B. 2015, ApJ, 812, 11

Vissers, G. J. M., van der Voort, L. H. M. R., \& Rutten, R. J. 2013, ApJ, 774,32

Wang, Y.-M. 2016, ApJL, 820, L13

Wang, Y.-M., Ugarte-Urra, I., \& Reep, J. W. 2019, ApJ, 885, 34

Watanabe, H., Vissers, G., Kitai, R., Rouppe van der Voort, L., \& Rutten, R. J. 2011, ApJ, 736, 71

Yang, H., Chae, J., Lim, E.-K., et al. 2013, SoPh, 288, 39

Young, P. R., Tian, H., Peter, H., et al. 2018, SSRv, 214, 120 